\title{
CESR LUMINOSITY DEPENDENCE ON MAGNETIC ALIGNMENT*
}

\author{
$\underline{\text { R. Holtzapple }}^{+}$and D. Rice \\ Laboratory of Nuclear Studies, Cornell University, Ithaca, NY 14853
}

\begin{abstract}
The Cornell electron-positron storage ring (CESR) luminosity has increased by a factor of $>3$ over the last 3 years with a peak luminosity of $8.0 \times 10^{32} \mathrm{~cm}^{-2} \mathrm{sec}^{-1}$ and a record integrated luminosity of $750 \mathrm{pb}^{-1}$ for one month. This increase in luminosity is in part due to improved alignment of magnetic elements. Operation with a pretzel [1] orbit in multiple bunch mode places particular importance on alignment because of the side effects of magnetic correctors. We report the improvements in alignment and estimate the effects on colliding beam performance.
\end{abstract}

\section{CESR LUMINOSITY}

While accurate alignment of magnetic elements is essential for good performance in all accelerators, the multi-bunch operation of CESR places additional demands on magnet alignment. Most of the two orders of magnitude increase in CESR luminosity since initial operation has been due to using many bunches in each beam [1].

The interaction between counter-rotating bunches, primarily through long range electromagnetic fields, must be limited at all parasitic (i.e., not intended for collisions) crossing points to avoid dilution of the beams' phase space density and resonance induced particle losses. Since only a single vacuum chamber is available in CESR the interaction is reduced by establishing separate closed orbits for the two counter rotating beams by means of electrostatic separators. These separate orbits follow large ( $\sim 15 \mathrm{~mm}$ peak) pseudo-sinelike betatron trajectories around the complete circumference.

Since the beams are far from the centerline of the magnets (with opposite displacements) for most of the circumference, sensitivity to nonlinear fields is increased and corrections are more difficult since electrons and positrons are affected differently. Horizontal-vertical coupling created by skew-quadrupole fields cannot be simply corrected by two appropriately placed skew quads since not only the coupling vector phases but also vertical betatron phases must be matched. Because of the vertical correctors' poor fringe field quality their strength must be limited for reliable beam-beam performance.

Increased alignment demands extend to vacuum chambers also since synchrotron radiation power can increase to dangerous levels when chambers are misaligned.

For a given ring and optics, luminosity may be quantified by two parameters - total beam current and beam-beam parameter, proportional to luminosity divided by a geometric mean of the two beam currents. The beam-beam parameter, $\xi$, is most directly affected by magnet alignment. Unintentional coupling, dispersion, and beta function errors directly affect $\xi$, as do unnecessarily strong correction magnets (e.g., to correct vertical orbit errors).

Effects on beam current will be less direct if an unrelated phenomenon limits currents. Otherwise resonances and dynamic aperture may reduce beam current due to magnet alignment errors.

\section{ALIGNMENT OF CESR}

There are 84 dipole and 102 quadrupole magnets in CESR. The vertical height, radial position, and rotation of the CESR quadrupoles are routinely measured and aligned to eliminate any unwanted orbit distortion of the CESR beam. Recently the dipole magnets have also been aligned as well. By aligning the quadrupole and dipole magnets in CESR we have reduced the dependence on steering magnets needed for orbit correction. Measurement of the magnetic elements in CESR is done with conventional techniques and is described in detail elsewhere $[2,3,4]$. In this paper a brief description of the method will be described and the accuracy of the measurement will be quoted.

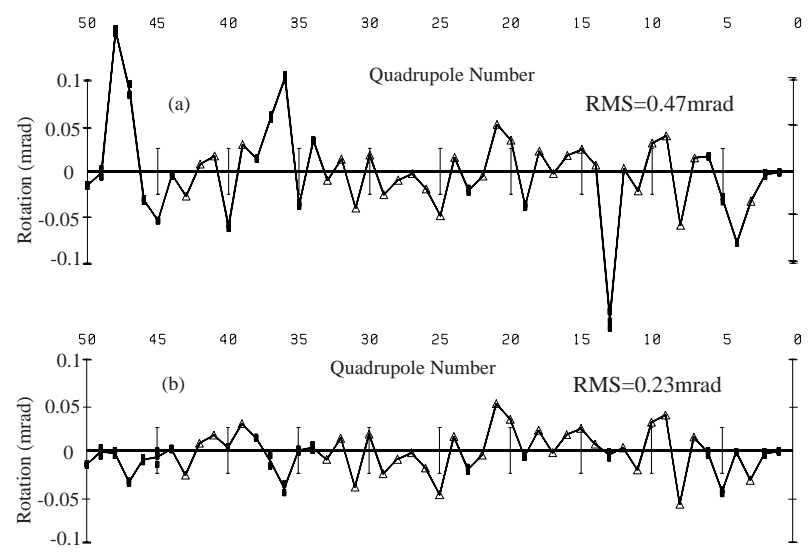

Figure 1. The rotation of the half of the quadrupole magnets in CESR (a) before alignment and (b) after alignment.

\footnotetext{
${ }^{*}$ Work supported by the National Science Foundation.

+Email:RLH@CESR10.LNS.Cornell.edu
} 
The vertical height of magnetic elements is measured using an optical level. This technique has a quadrupole to quadrupole accuracy of $\sim 5$ mils. The rotation (or level) of magnetic elements is measured using an electronic bar level. The bar level has an accuracy of $\sim 0.1 \mathrm{mrad}$. The radial measurement consists of wire measurements that relate each magnet to the magnets on either side. The radial positioning can be done to $\sim 20$ mils rms. The alignment data is analyzed using a series of computer programs that have been written specifically for CESR. Figure 1 is the rotation of the CESR quadrupole magnets before and after the magnets were aligned. It should be noted that when the CESR ring is aligned not all of the magnets are moved, only the ones that are outside of our prescribed tolerances.

\section{ORBIT MEASUREMENTS}

Several beam parameters have been measured to validate the usefulness of aligning the magnetic elements in CESR. They are (i) the zero corrector orbit, (ii) the coupling of CESR, and (iii) the corrector strengths during colliding beam physics. These quantities directly effect the performance of CESR by influencing the beam-beam tune parameter $\xi$. The following data were measured before and after quadrupole magnets were aligned in CESR and represent the type of improvement one would expect by aligning the quadrupole magnets.

The zero corrector orbit is the beam orbit with all steering correctors turned off. If all the magnetic elements were perfectly aligned with ideal fields then the zero corrector orbit would be zero and the beam would travel through the centers of the beam detectors and magnets. As an example, Figure 2 is the horizontal zero corrector orbits before and after the quadrupole magnets were aligned in CESR. The rms horizontal zero corrector orbit went from $5.30 \mathrm{~mm}$ to $4.52 \mathrm{~mm}$ by aligning the quadrupole magnets. We have not been as successful reducing the vertical zero corrector orbit. During the same quadrupole alignment period the rms vertical zero corrector orbit went from $5.56 \mathrm{~mm}$ to $5.60 \mathrm{~mm}$.
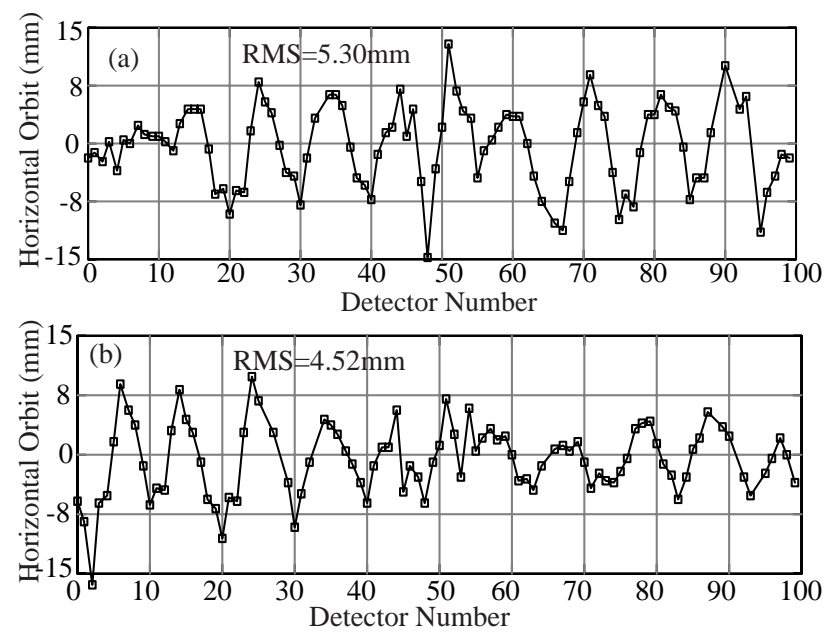

Figure 2. The CESR zero corrector horizontal orbit (a) before and (b) after aligning the quadrupole magnets.
Rotated quadrupoles introduce coupling between the horizontal and vertical planes which results in an increase in the vertical beam size and reduces the luminosity. A performance benefit that can be attributed to leveling the quadrupoles in CESR is the reduction of transverse coupling. To measure coupling in CESR a normal mode of the beam is coherently excited by a shaker and the relative phase and amplitude of the two normal transverse (vertical and horizontal) modes is measured [5]. From this measurement the coupling transfer matrix $\bar{C}$ can be determined. The $\overline{\mathrm{C}}_{12}$ term of the $2 \times 2$ coupling matrix was measured before and after the quads were leveled and the results are shown in Figures 3.

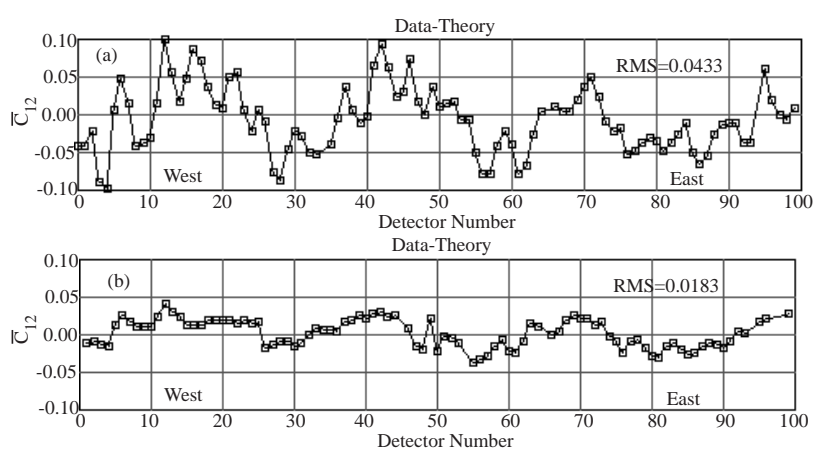

Figure 3. $\bar{C}_{12}$ at each detector around the ring measured (a) before and (b) after the quadrupoles were leveled. The rms. value is $4.3 \%$ before and $1.8 \%$ after the quadrupoles were aligned [6].

Several comments can be made about the coupling results. 1) The coupling measurements shown were made with the skew quadrupoles turned off so the two measurements are on equal footing as far as coupling goes. 2) During the down period the back-leg windings of some of the dipole steering were moved away from the beam-pipe and that might have reduced the coupling in addition to leveling the quadrupoles. 3) Both measurements have significant global coupling (long wavelength) but the localized coupling (short wavelength) is greatly reduced after the quadrupole magnets were leveled.
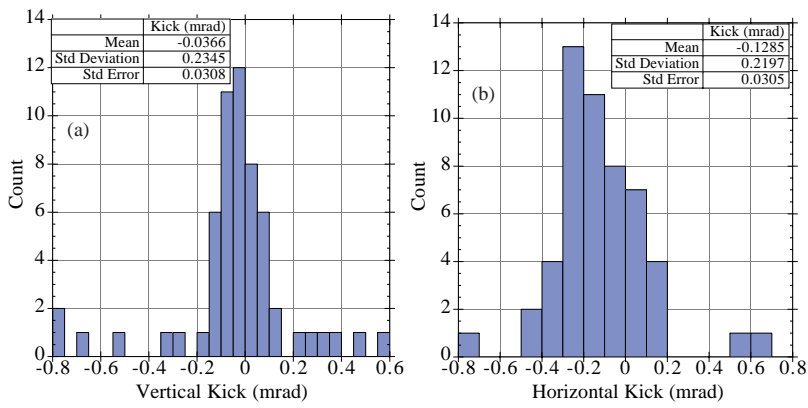

Figure 4. The (a) vertical and (b) horizontal corrector strength for during collisions before the magnetic elements were aligned in CESR.

Vertical and horizontal corrector magnets steer the beam to an ideal orbit tuned for luminosity. The pretzel orbit used in multiple bunch operation has the beam far 
from centered in these steering magnets. For the steering coils, nonlinear fields increase with displacement and reduce the dynamic aperture of CESR. It is therefore imperative to rely on these corrector magnets as little as possible. Aligning magnetic elements in CESR reduces the dependence on corrector magnets. The corrector strengths during colliding beams were measured before and after the magnets were aligned (Figures 4 and 5).
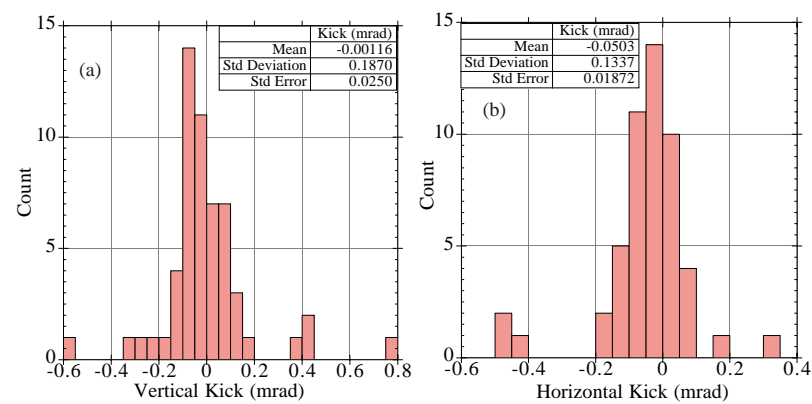

Figure 5. The (a) vertical and (b) horizontal corrector strength during high energy physics collisions after the magnetic elements were aligned in CESR.

The reduction in the standard deviation of the corrector strength was by $25 \%$ for vertical correctors and $64 \%$ for horizontal correctors.

These results prove the usefulness of aligning the magnetic elements in CESR.

\section{LUMINOSITIES DEPENDENCE ON MAGNETIC ALIGNMENT}

The luminosity of an electron-positron storage ring is

$$
\mathrm{L} \approx \frac{\mathrm{f}_{0} \mathrm{~N} \gamma \xi}{2 \mathrm{r}_{\mathrm{e}} \beta_{\mathrm{V}}^{*}}
$$

where $\mathrm{N}$ is the number of particles per bunch, $\mathrm{f}_{0}$ is the revolution frequency, $r_{\mathrm{e}}$ is the classical electron radius, $\xi$ is the beam-beam parameter, $\gamma=\mathrm{E} / \mathrm{mc}^{2}$, and $\beta_{\mathrm{V}}^{*}$ is the vertical beta function at the interaction point. The luminosity is directly proportional to the beam-beam parameter and we will try to correlate the increase in the beam-beam parameter, and hence luminosity, with magnetic alignment.

The beam-beam parameter is recorded during CESR beam collisions. Figure 6 is the average peak beam-beam for each running cycle from November of 1992 to February of 1999. The schedule for colliding beams at CESR consists of running periods usually 10 to 20 weeks in length followed by down periods for improvements and maintenance. Each data point is the average beam-beam parameter for that running period. The arrows denote the down periods when significant alignment of magnetic elements occurred with the expectation of an improvement to CESR performance. Several interesting features should be pointed out from Figure 6. 1) The beam-beam parameter on average goes up by $11 \pm 6 \%$ when the magnetic elements are aligned during the down period. 2) The beam-beam parameter goes down on average by $4 \pm 5 \%$ when the magnetic elements are not aligned during the down period. 3) If magnets are not aligned over several running periods the beam-beam parameter tends to suffer. It should be noted that during each downperiod other modifications of CESR are made. The gain in the beam-beam parameter is not solely due to magnetic alignment.

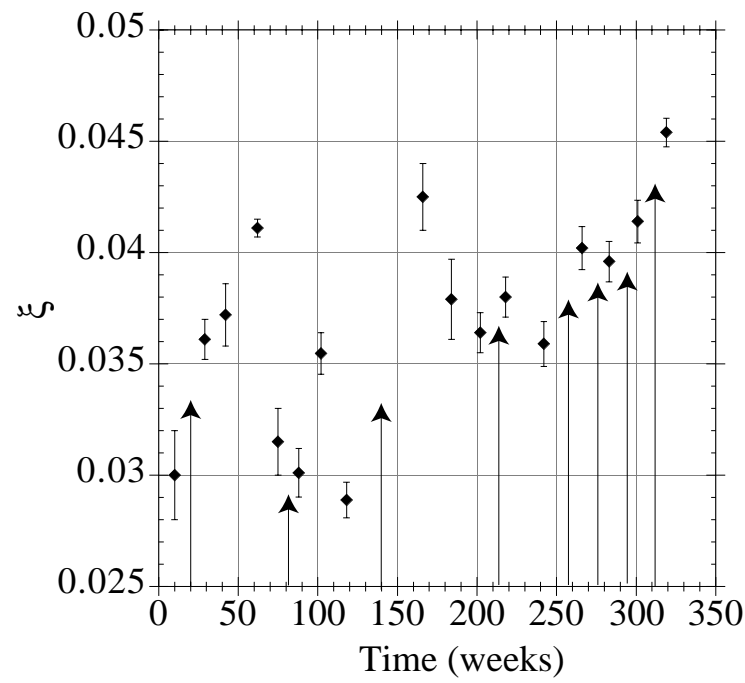

Figure 6. The mean peak beam-beam parameter from November 1992 to February 1999. The arrows indicate a downperiod when the CESR magnetic elements were aligned.

\section{CONCLUSION}

Aligning the magnetic elements in CESR reduces the dependence on steering correctors, reduces coupling, and increases the beam-beam parameter and, on average, increases the delivered luminosity of CESR. We have noted an increase in luminosity of $\sim 11 \%$ due to magnetic alignment of CESR.

\section{ACKNOWLEDGEMENTS}

The authors would like to thank David Sagan for taking the coupling measurements and the survey and alignment group (SAG) for an excellent job of aligning the magnets. Gerry Rouse, who has lead the survey group for the past ten years, will be greatly missed.

\section{REFERENCES}

[1] Littauer R., "Multibunch Operation of CESR", IEEE NS-32, 1610 (1985).

[2] Holtzapple R., et. al., "Radial Alignment of the Quadrupole Magnets in the Cornell Electron-Positron Storage Ring", to be published

[3] Holtzapple R., et. al., "Alignment of the Dipole Magnets in the Cornell Electron-Positron Storage Ring”, CBN-98-22

[4] Holtzapple R., et. al., "Leveling of the Quadrupole Magnets in the Cornell Electron-Positron Storage Ring", To be published.

[5] Bagley, P. and Rubin, D.,"'Correction of Transverse Coupling in a Storage Ring", Proc. 1989 Part. Acc. Conf. (San Francisco) p. 874, 1989.

[6] The CESR coupling measurements are provided by David Sagan. 